\title{
Frailty Prevalence and Characteristics in Older Patients with Type 2 Diabetes
}

\author{
Takahisa Tanikawa ${ }^{*}$, Sayuri Sable-Morita², Haruhiko Tokuda3 ${ }^{3}$, Hidenori Arai ${ }^{4}$ \\ ${ }^{1}$ Department of Endocrinology, National Center for Geriatrics and Gerontology, Obu, Japan \\ ${ }^{2}$ Nursing Department, National Center for Geriatrics and Gerontology, Obu, Japan \\ ${ }^{3}$ Department of Clinical Laboratory, National Center for Geriatrics and Gerontology, Obu, Japan \\ ${ }^{4}$ National Center for Geriatrics and Gerontology, Obu, Japan \\ Email: ^hoisan@ncgg.go.jp
}

How to cite this paper: Tanikawa, T., Sable-Morita, S., Tokuda, H. and Arai, H. (2019) Frailty Prevalence and Characteristics in Older Patients with Type 2 Diabetes. Journal of Diabetes Mellitus, 9, 31-38. https://doi.org/10.4236/jdm.2019.92004

Received: March 3, 2019

Accepted: May 3, 2019

Published: May 6, 2019

Copyright (c) 2019 by author(s) and Scientific Research Publishing Inc. This work is licensed under the Creative Commons Attribution International License (CC BY 4.0).

http://creativecommons.org/licenses/by/4.0/

\begin{abstract}
Background: Older diabetic patients are more likely to be frail than those who do not have diabetes. Frailty is an important risk factor for both mortality and disability in older patients with type 2 diabetes. However, the mechanism of frailty in diabetes mellitus is not fully understood. Aims: The aim of this study was to identify the prevalence of frailty and associated factors in older patients with type 2 diabetes in Japan. Methods: A cross-sectional study was conducted with a total of 178 outpatients who were over 65 years old with type 2 diabetes. We used the Obu Study Health Promotion for the Elderly definition of frailty to divided subjects into a non-frail and a frail group. We investigated the association between frailty and various patient characteristics. Results: In the study, $21.4 \%$ of the older patients with type 2 diabetes were considered frail. There were no significant differences in the duration of diabetes, BMI, proportion of microvascular complications, or $\mathrm{HbA} 1 \mathrm{c}$ values between the frail and non-frail group. However, serum albumin and IGF-1 levels were lower in the frail group than the non-frail group as were the Mini-Mental State Examination scores. The frail group had a higher number of medications than the non-frail group. In a multivariable analysis, frailty was positively associated with the number of medications and, lower levels of both serum albumin and IGF-1. Conclusion: Our study suggests that diabetes accelerates the aging process and frailty is associated with low albumin, polypharmacy and low levels of IGF-1.
\end{abstract}

\section{Keywords}

Frailty, Albumin, Insulin-Like Growth Factor-1, Polypharmacy 


\section{Introduction}

The prevalence of type 2 diabetes is increasing in the older population of Japan with patients over 65 years of age accounting for $40 \%$ or more of all diabetes patients [1]. Frailty is a state of increased vulnerability to stressors with reduced ability to maintain homeostasis. This condition is highly prevalent in old age and increases the risk of adverse outcomes, including falls, disability, hospitalization, and mortality [2] [3]. Identification of frailty is important as interventions may prevent or delay its clinical consequences. Older diabetic patients are more likely to be frail than those who do not have diabetes [4] [5]. The prevalence of frailty in a population of people with type 2 diabetes varies and has reported being between $5 \%-48 \%$, based on different diagnostic criteria [6]. Frailty is an important risk factor for both mortality and disability in older patients with type 2 diabetes [7]. Several reports suggest that the assessment of frailty must become part of the routine evaluation of older diabetic patients [7] [8] [9]. However, the connection between diabetes and frailty is not fully understood in a clinical setting.

The purpose of this study was to identify the prevalence of frailty and factors associated with frailty in older patients with type 2 diabetes in Japan.

\section{Methods}

\subsection{Participants}

A cross-sectional study was conducted with a total of 178 patients who were over the age of 65 years, who had type 2 diabetes, and were attending an outpatient diabetes clinic of the National Center for Geriatrics and Gerontology between August 2015 to April 2016. Patients were selected randomly. If the total Barthel index, which measures functional independence, was $<90$ or 0 in any domains, the individual was considered to be disabled [10] and was excluded from the study. Individuals with acute disease or unstable chronic disease were also excluded.

The ethics committee of the National Center for Geriatrics and Gerontology approved the study protocol. We carried out this study in accordance with the provisions of the Declaration of Helsinki. Written informed consent was obtained from all participants before the study began.

\subsection{Measurements}

Medical records were used to obtain participants' age, duration of disease, blood test results, general physical measurements, number of current medications and diabetic complications. BMI was calculated as weight in kilograms divided by squared height in meters $\left(\mathrm{kg} / \mathrm{m}^{2}\right)$.

The concentrations of hemoglobin $(\mathrm{Hb})$, serum albumin, creatinine, LDL-cholesterol (LDL-C), HDL-cholesterol (HDL-C), triglyceride, aspirate aminotransferase (AST), alanine aminotransferase (ALT), and HbAlc were measured using automated techniques at the central laboratory of our hospital. 
The estimated glomerular filtration ratio (eGFR) was calculated using the serum creatinine concentration. Serum growth hormone (GH) and IGF-1 were assessed using immunoradiometric assay.

\subsection{Frailty Evaluation}

We evaluated frailty using the Obu Study of Health Promotion for the Elderly frailty scale, which included the following 5 components: slowed walking speed, reduced grip strength, low endurance, physical inactivity, and poor nutrition [11]. Each domain yielded a dichotomous score of 0 if the participant did not have these symptoms or 1 if the following criteria were met:

1) Slowed walking speed was established according to a cutoff of $<1.0 \mathrm{~m} / \mathrm{s}$. Walking speed was defined as the speed at which a patient could walk a distance of $2.4 \mathrm{~m}$.

2) Weakness was assessed using grip strength. Low grip strength was established according to a sex-specific cutoff (male: $<26 \mathrm{~kg}$, female: $<17 \mathrm{~kg}$ ).

3) Endurance was assessed via a self-repot of exhaustion, which included questions from the Geriatric Depression Scale, such as "Do you feel full of energy?".

4) Physical activity was assessed using the following questions: "Do you engage in moderate levels of physical exercise or sports aimed at health?" and "Do you engage in low levels of physical exercise aimed at health?”. If participants answered "no" to both questions, they were considered physically inactive.

5) Nutritional status was determined according to self-reports of weight loss. Weight loss was defined as unintentional weight loss of $\geq 5 \%$ of total body weight in the past 2 years.

An ordinary variable with scores ranging from 0 to 5 was then created to account for the total points of all the above five components. We defined participants with $\geq 3$ points as frail.

\subsection{Statistical Analysis}

Continuous data were tested for normal distribution by the Kolmogorov-Smirnov test. The data were presented as mean \pm standard deviations (SD) in the case of normally distributed data and as the median and interquartile range (IQR) in the case of non-normally distributed data. To compare population characteristics by the frailty status, either unpaired Student's $t$ test or Mann-Whitney $U$ test was used for continuous variables, depending on the distribution of the variables. The Pearson chi-squared test was used for categorical variables. Binary logistic regression was used to investigate cross-sectional associations between frailty status and specific variables. Odds ratios and 95\% CIs are reported for logistic regression analysis and $p$-values less than 0.05 were considered statistically significant. Data were analyzed using the Statistical Package for the Social Sciences (SPSS ${ }^{\circledR}$ ) for Windows, version 23.0 (IBM Corporation, Chicago, IL). 


\section{Results}

Of the 178 older patients with type 2 diabetes, 38 (21.4\%) were frail (Table 1 ). Table 2 shows the overall clinical characteristics for the 178 patients, and the clinical characteristics for the group with frail and non-frail group. The median age and number of medications were higher in the frail group, while the median levels of serum albumin, GH and IGF-1 were lower in the frail group than those in the non-frail group. The frail group also showed a lower median Min-Mental State Examination scores. While the proportion of dyslipidemia is lower in the frail groups, the levels of LDL-C, HDL-C and TG were not significant differences. Between the frail and non-frail groups, there were no significant differences in the duration of diabetes, BMI, proportion of microvascular complications and HbA1c levels. There were no differences between the two groups in of the classes or types of medications used (Data not shown). Multivariable logistic regression analysis was performed to evaluate associations with frailty. After adjustment for age and sex, the multiple regression analysis indicated that a higher number of medications and, low levels of both serum albumin and IGF-1 were significantly associated with frailty (Table 3 ).

Table 1. Prevalence of frailty.

\begin{tabular}{ccccc}
\hline & Total & Non-frail & Frail & P \\
\hline $\mathrm{N}(\%)$ & $178(100)$ & $140(78.6)$ & $38(21.4)$ & \\
Age (years) & $74(70-78)$ & $73.5(69.8-77.3)$ & $78.5(74-83)$ & $\mathrm{P}<0.05$ \\
Male (\%) & $92(51.7)$ & $72(51.4)$ & $20(52.6)$ & 1 \\
\hline
\end{tabular}

Data in the table are presented as whole number (percentage) or median (IQR) as appropriate.

Table 2. Clinical characteristics of 178 participants and comparison of various factors between the frail or non-frail groups.

\begin{tabular}{ccccc}
\hline & Total & Non-frailty & Frailty & P \\
\hline Duration (years) & $14.1(19.8-9)$ & $12(9-19)$ & $13.5(10-20)$ & 0.351 \\
BMI (kg/m²) & $23.8(21.4-26.4)$ & $23.9(21.7-26.2)$ & $23.1(20.8-26.7)$ & 0.576 \\
MMSE (points) & $28(25-29)$ & $28(26-30)$ & $26(23-30)$ & P $<0.05$ \\
Medications (n) & $6(4-8)$ & $5(3-7.3)$ & $8(6-9)$ & P $<0.05$ \\
Insulin (\%) & $44(24.7)$ & $33(22.9)$ & $12(31.6)$ & 0.292 \\
Peripheral neuropathy (\%) & $92(51.7)$ & $67(47.9)$ & $25(65.8)$ & 0.0667 \\
Retinopathy (\%) & $57(32)$ & $42(29.3)$ & $16(42.1)$ & 0.17 \\
Nephropathy (\%) & $109(61.2)$ & $82(57.6)$ & $27(71.1)$ & 0.191 \\
Hypertension (\%) & $111(62.4)$ & $84(60)$ & $27(71.1)$ & 0.259 \\
Dyslipidemia (\%) & $137(77.0)$ & $114(81.4)$ & $23(60.5)$ & P $<0.05$ \\
CAD (\%) & $35(19.7)$ & $26(18.6)$ & $9(23.7)$ & 0.494 \\
CVD (\%) & $15(8.43)$ & $10(7.1)$ & $5(13.2)$ & 0.319 \\
PAD (\%) & $23(12.9)$ & $15(10.7)$ & $81.1)$ & 0.105 \\
\hline
\end{tabular}




\section{Continued}

\begin{tabular}{ccccc}
\hline Albumin (g/dl) & $4.3(4.1-4.6)$ & $4.4(4.2-4.6)$ & $4.1(3.8-4.5)$ & $\mathrm{P}<0.05$ \\
eGFR (ml/min./1.73m²) & $64.6 \pm 18.9$ & $64.8 \pm 17.8$ & $63.9 \pm 22.9$ & 0.798 \\
GOT (U/L) & $22(18-28)$ & $22(18-28)$ & $22.5(18-26)$ & 0.798 \\
GPT (U/L) & $20(15-26)$ & $20(15-27)$ & $22.5(18-26)$ & 0.435 \\
Hb (g/dl) & $13.6(12.6-14.6)$ & $13.6(12.9-14.5)$ & $13(12.1-14.7)$ & 0.327 \\
HbAlc (\%) & $7.2(6.7-8.0)$ & $7.2(6.7-7.9)$ & $7.6(6.8-8.3)$ & 0.12 \\
HDL-C (mg/dl) & $56(47-65)$ & $56(46.5-65)$ & $57(48-66.8)$ & 0.554 \\
LDL-C (mg/dl) & $112(94-133)$ & $112(95-133)$ & $110(89.3-130.5)$ & 0.339 \\
TG (mg/dl) & $124(85-162)$ & $126(88-165.5)$ & $116.5(85-162)$ & 0.718 \\
GH (ng/ml) & $0.3(0.09-0.87)$ & $0.22(0.07-0.69)$ & $0.78(0.21-1.5)$ & $\mathrm{P}<0.05$ \\
IGF-1 (ng/ml) & $132 \pm 49.5$ & $140.1 \pm 46.0$ & $101.1 \pm 50.2$ & $\mathrm{P}<0.05$ \\
\hline
\end{tabular}

CAD, coronary artery disease; CVD, cerebrovascular disease; PAD, peripheral artery disease. Data in the table are presented as whole number (percentage), mean $\pm \mathrm{SD}$, or median (IQR) as appropriate.

Table 3. Odds ratio (95\% confidence interval) for the association between several factors and frailty.

\begin{tabular}{cccc}
\hline & OR & $95 \%$ CI & $\mathrm{P}$ \\
\hline Albumin & 0.24 & 0.084 & $\mathrm{P}<0.05$ \\
IGF-1 & 0.988 & $0.978-0.997$ & $\mathrm{P}<0.05$ \\
Number of medications & 1.2 & $1.05-1.37$ & $\mathrm{P}<0.05$ \\
\hline
\end{tabular}

OR, odds ratio; CI, confidence intervals. Logistic regression model adjusted for age and sex.

\section{Discussion}

In a previous study, the prevalence of frailty was found to be $11.3 \%$ for community-dwelling people in the same area, using the same definition of frailty [11]. The prevalence of frailty was higher in this study which suggests diabetes may accelerate the aging process.

In the present study, we found that the following factors were associated with frailty: hypoalbuminemia, increased number of medications, and low levels of IGF-1. Serum albumin levels correlate inversely with functional decline in older people [12]. Hypoalbuminemia is the result of the combined effects of inflammation and inadequate protein and caloric intake in patients with chronic disease [13]. Low energy intake or low nutrient intake is significantly associated with frailty [14]. Therefore, hypoalbuminemia is thought to be related to undernutrition in and frailty in patients with diabetes mellitus.

IGF-1 plays important roles in the regulation of growth and cellular proliferation, and its synthesis is stimulated by nutrition and GH in liver and other tissues. Since IGF-1 is also regulated by in the liver, a significant reduction was observed in patients with type 1 diabetes [15] [16]. In type 2 diabetes, the levels of IGF-1 varied across several studies [17] [18]. Serum IGF-1 levels may be dependent on the balance between insulin resistance and insulin secretion in type 2 diabetes. In our study serum IGF-1 levels in the frail group were lower than the 
non-frail group. Doi et al. reported that a lower serum IGF-1 level is independently related to disability among older people [19].

In our study the number of medications in the frail group was greater than in the non-frail group. Although there is no consensus on the exact definition, concurrent use of multiple medications is known as polypharmacy. Patients with diabetes are often on multi-drug regimens which are needed for tight glycemic control, and frequently have comorbidities which also require multiple medications. Polypharmacy is associated with a higher incidence of frailty in older people [20]. While comorbidities are often seen in older populations, two studies found that polypharmacy was associated with frailty by using statistical analyses that controlled for multiple potential confounders including comorbidity [20] [21].

In our study the levels of $\mathrm{HbAlc}$ were not significantly different between the non-frail group and frail group. There has not been a definitive answer on the relationship as different studies have shown disparate results. One previous study showed hyper glycemia is associated with the development of frailty [22], while another reported that low glycated hemoglobin levels were associated with higher severity of frailty defined by using the Clinical Frailty Scale (CFS) [1]. In the study of Zaslavsky and colleagues, average glucose levels less than $160 \mathrm{mg} / \mathrm{dl}$ and greater than $180 \mathrm{mg} / \mathrm{dl}$ were related to an increased risk of frailty. This showed an apparent $\mathrm{U}$-shape association between glucose levels and frailty in diabetes patients [23]. Since our study is a cross sectional study and HbAlc is the average blood glucose levels for the last two to three months, there may be no significant differences between the frail and non-frail group.

\section{Conclusion}

In conclusion, we have documented the prevalence of frailty in older patients with type 2 diabetes and indicated that undernutrition, polypharmacy and low IGF-1 levels are associated with frailty. Our study had several limitations, including the cross-sectional design. Further longitudinal research studies are needed to identify the underlying mechanisms for these associations.

\section{Acknowledgements}

This study was supported by the Research Fund Science (27-9) from the National Center for Geriatrics and Gerontology (NCGG), Japan.

\section{Author Contributions}

T.T researched the data and wrote the manuscript, S.S researched the data, and H.T. and H.A. reviewed and edited the manuscript. T.T is the guarantor of this work and, as such, had full access to all the data in the study and takes responsibility for the integrity of the data and the accuracy of the data analysis. All authors read and approved the final manuscript. 


\section{Conflicts of Interest}

No potential conflict of interest relevant to this article was reported.

\section{References}

[1] Ynagita, I., Fujiwara, Y., Eda, T., Tajima, M., Yonemura, K., Kawajiri, T., Yamaguchi, N., Asakawa, H., Nei, Y., Kayashima, Y., Yoshimoto, M., Kitajima, Y., Harada, M., Araki, Y., Yoshimonoto, S., Aida, Y., Yoshimoto, S., Aida, E., Yanase, T., Nawata, H. and Muta, K. (2018) Low Glycated Hemoglobin Level Is Associated with Severity of Frailty in Japanese Elderly Diabetes Patients. Journal of Diabetes Investigation, 9, 419-425. https://doi.org/10.1111/jdi.12698

[2] Fried, L.P., Tangen, C.M., Walston, J., Newman, A.B., Hirsch, C., Gottdiener, J., Seeman, T., Tracy, R., Kop, W.J., Burke, G. and McBumie, M.A. (2001) Frailty in Older Adults: Evidence for Phenotype. The Journals of Gerontology. Series A, Biological Sciences and Medical Sciences, 56, M146-M156. https://doi.org/10.1093/gerona/56.3.M146

[3] Ensrud, K.E., Ewing, S.K., Taylor, B.C., Fink, H.A., Cawthon, P.M., Stone, K.L., Hiller, T.A., Cauley, J.A., Hochberg, M.C., Rodondi, N., Tracy, J.K. and Cumming, S.R. (2008) Comparison of 2 Frailty Indexes for Prediction of Falls, Disability, Fractures, and Death in Older Women. Archives of Internal Medicine, 168, 382-389. https://doi.org/10.1001/archinternmed.2007.113

[4] Hubbard, R.E., Andrew, M.K., Fallah, N. and Rockwood, K. (2010) Comparison of the Prognostic Importance of Diagnosed Diabetes, Co-Morbidity and Frailty in Older People. Diabetic Medicine, 27, 603-606.

https://doi.org/10.1111/j.1464-5491.2010.02977.x

[5] Cacciatore, F., Testa, G., Galizia, G., Della-Morte, D., Mazzella, F., Langellotto, A., Pirozzi, G., Ferro, G., Gargiulo, G., Ferrara, N., Rengo, F. and Abete, P. (2013) Clinical Frailty and Long-Term Mortality in Elderly Subjects with Diabetes. Acta Diabetologica, 50, 251-260. https://doi.org/10.1007/s00592-012-0413-2

[6] Perkisas, S. and Vandewoude, M. (2016) Where Frailty Meets Diabetes. Diabetes/Metabolism Research and Reviews, 32, 261-267.

https://doi.org/10.1002/dmrr.2743

[7] Castro-Rodriguez, M., Carnicero, A., Garcia-Garcia, F.J., Walter, S., Morley, J.E., Rodriguez-Artalejo, F., Sinclair, A.J. and Rodrigguez-Manas, L. (2016) Frailty as a Major Factor in the Increased Risk Death and Disability in Older People with Diabetes. JAMDA, 17, 949-995. https://doi.org/10.1016/j.jamda.2016.07.013

[8] Strain, W.D., Hope, S.V., Green, A., Kar, P., Valabhi, J. and Sinclair, A.J. (2018) Type 2 Diabetes Mellitus in Older People: A Brief Statement of Key Principles of Modern Day Management Including the Assessment of Frailty. A National Collaborative Stakeholder Initiative. Diabetic Medicine, 35, 834-845. https://doi.org/10.1111/dme.13644

[9] Sinclair, A.J., Abdelhafiz, A., Dunning, T., Izuquirdo, M., Rodriguez, M.L., Bourdel-Marchasson, M.J.E., Munshi, M., Woo, J. and Vellas, B. (2018) An International Position Statement on the Management of Frailty in Diabetes Mellitus: Summary of Recommendations. The Journal of Frailty \& Aging, 7, 10-20.

[10] Mahoney, F.I. and Barthel, D.W. (2001) Functional Evaluation: The Barthel Index. Maryland State Medical Journal, 14, 61-65.

[11] Shimada, H. (2013) Combined Prevalence of Frailty and Mild Cognitive Impairment in a Population of Elderly Japanese People. JAMDA, 14, 518-524. 
https://doi.org/10.1016/j.jamda.2013.03.010

[12] Hubbard, R.E., O’Mahony, M.S., Savva, G.M., Calver, B.L. and Woodhouse, K.W. (2009) Inflammation and Frailty Measures in Older People. Journal of Cellular and Molecular Medicine, 13, 3103-3109. https://doi.org/10.1111/j.1582-4934.2009.00733.x

[13] Don, B.R. and Kaysen, G. (2004) Serum Albumin: Relationship to Inflammation and Nutrition. Seminars in Dialysis, 17, 432-437. https://doi.org/10.1111/j.0894-0959.2004.17603.x

[14] Bartali, B., Frongillo, E.A., Bandinelli, S., LauretanSemba, R.D., Fried, L.P. and Ferrucci, L. (2006) Low Nutrient Intake Is an Essential Component of Frailty in Older Persons. Journal of Gerontology, 61, 589-593. https://doi.org/10.1093/gerona/61.6.589

[15] Boni-Schnetzler, M., Schmid, C., Meier, P.J. and Froesch, E.R. (1991) Insulin Regulates Insulin-Like Growth Factor 1 mRNA in Rat Hepatocytes. American Journal of Physiology, 260, E846-E851. https://doi.org/10.1152/ajpendo.1991.260.6.E846

[16] Paluta, M., LeCaire, T., Sadek-Badawi, M., Herrera, V. and Danielson, K.K. (2014) The Trajectory of IGF-1 across Age and Duration of Type 1 Diabetes. Diabetes/Metabolism Research and Reviews, 30, 777-783.

https://doi.org/10.1002/dmrr.2554

[17] Frystyk, J., Skjaerbaek, C., Vestbo, E., Fisker, S. and Orskov, H. (1999) Circulating Levels of Free Insulin-Like Growth Factors in Obese Subjects: The Impact of Type 2 Diabetes. Diabetes/ Metabolism Research and Reviews, 15, 314-322. https://doi.org/10.1002/(SICI)1520-7560(199909/10)15:5<314::AID-DMRR56>3.0.C $\underline{\mathrm{O} ; 2-\mathrm{E}}$

[18] Rajpathak, S.N., He, M., Sun, Q., Kaplan, R.C., Muzumdar, R., Rohan, T.E., gunter, M.J., Pollak, M., Kim, M., Pessin, J.E., Wylie-Rosett, J., Hu, F.B. and Strickler, H.D. (2012) Insulin-Like Growth Factor Axis and Risk of Type 2 Diabetes Women. Diabetes, 61, 2248-2254. https://doi.org/10.2337/db11-1488

[19] Doi, T., Shimada, H., Makizako, H., Tsutsumimoto, K., Hotta, R., Nakakubo, S. and Suzuki, T. (2016) Insulin-Like Growth Factor-1 Related to Disability among Older Adults. The Journals of Gerontology. Series A, Biological Sciences and Medical Sciences, 71, 797-802. https://doi.org/10.1093/gerona/glv167

[20] Veronese, N., Stubbs, B., Nolae, M., Soimi, M., Pilotto, A., Vaona, A., Demurtas, J., Mueller, C., Huntley, J., Crepaldi, G. and Maggi, S. (2017) Polypharmacy Is Associated with Higher Frailty Risk in Older People: An 80 Year Longitudinal Cohort Study. JAMDA, 18, 624-628. https://doi.org/10.1016/j.jamda.2017.02.009

[21] Saum, K.U., Schöttker, B., Meid, A.D., Holleczek, B., Haefeli, W.H., Hauer, K. and Bernner, H. (2017) Is Polypharmacy Associated with Frailty in Older People? Results from the ESTHER Cohort Study. Journal of the American Geriatrics Society, 65, e27-e32. https://doi.org/10.1111/jgs.14718

[22] Kalyani, R.R., Tian, J.T., Xue, Q.L., Walston, J., Cappola, A.R., Fried, L.P., Brancati, F.L. and Blaum, C.S. (2012) Hyperglycemia and Incidence of Frailty and Lower Extremity Mobility Limitations in Old Women. Journal of the American Geriatrics Society, 60, 1701-1707. https://doi.org/10.1111/j.1532-5415.2012.04099.x

[23] Zaslavsky, O., Walker, R.L., Crane, P.K., Gray, S.L. and Larson, E.B. (2016) Glucose Levels and Risk of Frailty. The Journals of Gerontology. Series A, Biological Sciences and Medical Sciences, 71, 1223-1229.

https://doi.org/10.1093/gerona/glw024 